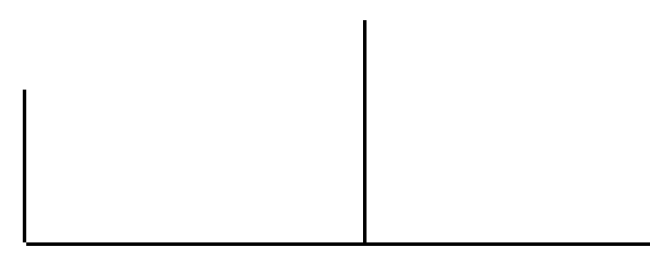

Rev. Latinoam. Psicopat. Fund., IX, 4, 636-646

\title{
Les objets de la pulsion et la construction de l'object psychique chez l'adolescent déliquant*
}

\author{
Rosane de Abreu e Silva
}

\begin{abstract}
Tout au long du développement infantile, l'être humain doit se former des représentations des objets de la pulsion à l'intérieur de la psyché. L'adolescence ne fait que montrer les effets des premières expériences vécues, en particulier les possibilités de se construire um objet psychique. La dialectique entre le dedans et le dehors, entre le sujet et l'objet inscrite avec les premiers objets de la pulsion, est au cœur de la pathologie de la délinquance juvénile.
\end{abstract}

Mots clés: Objets de la pulsion, objet psychique, adolescence, délinquance

* Cet article se fonde sur la thèse La délinquance juvénile et la question de l'objet présentée et soutenue à l’Université Paris 13, le 05 février 2004, sous la direction du Professeur Jean-Jacques Rassial. 


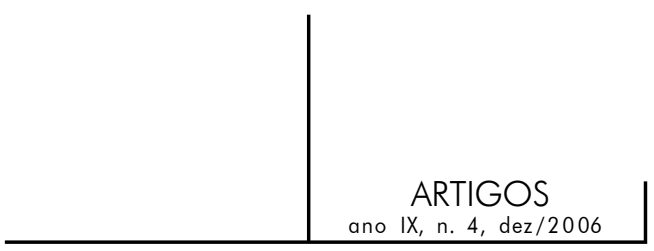

Les objets de la pulsion dans la période infantile et leurs remaniements à l'adolescence

L'emploi du terme objet en psychanalyse prend son origine dans la théorie de Freud (1915), lorsqu'il analyse la notion de pulsion et y distingue l'objet et le but. À l'origine, il affirme que l'objet de la pulsion est l'élément le plus variable. Il est la voie par laquelle la pulsion peut atteindre son but, donc le moyen contingent de la satisfaction. Dans la pensée freudienne (1905), l'objet de la pulsion prend son axe sur le fonctionnement de la sexualité infantile et sur les avatars de la sexualité pubertaire, où la vie sexuelle va désormais s'orienter définitivement sur autrui. De même, si l’on conçoit une évolution de l'objet pulsionnel qui aboutit à un objet d'amour génital se définissant par son autonomie et son caractère de totalité, il est nécessairement en relation avec l'édification progressive de la perception de l'objet.

Dans cette perspective du développement, nous pouvons inférer que le sujet passera d'une phase à l'autre par une intégration progressive de ses pulsions partielles, jusqu'à aboutir au sein de l'organisation génitale. Les premières pulsions sont dites partielles car leur satisfaction est liée à une zone érogène déterminée et surtout à ce que la théorie psychanalytique appelle objets partiels. En fait, ces objets dits partiels se situent à la limite entre le Moi et le non-Moi et ils ne sont source de plaisir que par leur rapport très particulier au corps propre pour lequel le corps de l'Autre reste toujours le point de référence essentiel. Entre ces objets s'établissent des échanges qui font que la vie pulsionnelle passe par une série d'avatars.

En somme, une transformation doit se produire dans le rapport au premier objet. La Mère primordiale doit disparaître en laissant la place à la Chose dans le psychisme. Lacan (1959-1960) l'articule au principe de plaisir: “Das Ding”. La Chose est du côté de la sublimation, elle fonde l'orientation du sujet vers l'objet, l'impossibilité de la jouissance pleine, à savoir qu'il n'y a pas de rencontre avec un objet absolu du désir. Le seul recours possible pour le sujet sera justement la quête de nouveaux objets. 


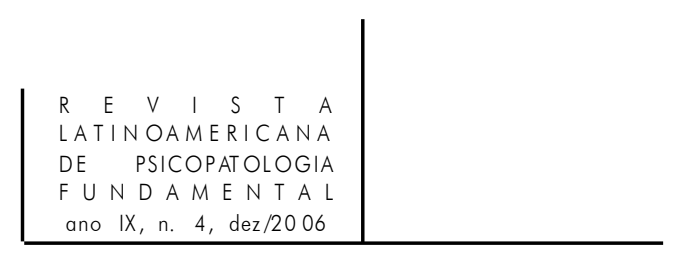

En conséquence, il doit y avoir un passage ouvert: celui de la symbolisation. Les objets partiels visés par l'activité pulsionnelle doivent se détacher de l'Autre maternel dans une mesure symbolique. D’après Penot (2000), la corporéité originaire des objets premiers de la pulsion, qui relèvent de la catégorie psychique du réel, forme inévitablement des résidus dans le processus de sublimation, et ce en tant que traces inconscientes qui tendront à susciter des formes toujours approximatives de représentation de ces objets. Il est important de remarquer également que Freud (1938) évoque la mère comme "l'objet du premier et du plus puissant des amours” (p. 60). Si au départ Freud conçoit l'objet comme ce qu'il y a de plus variable dans la pulsion, sa conception se déplace ensuite de l’objet variable à un seul objet.

Par conséquent, la première expérience vis-à-vis de l’objet pulsionnel construit les bases de la relation du Moi à l'objet. Le stade purement narcissique - dans lequel l'objet n'était pas différencié - doit être relayé par le stade d'objet, où plaisir et déplaisir signifient relation du Moi à l'objet. Ce dernier étant la source de plaisir, il y aura une tendance à le rapprocher et à l'incorporer. Par contre, lorsque l'objet est source de sensations de déplaisir, une tendance s'efforce de l'éloigner du moi. Le jeune enfant ressent une répulsion de l'objet et le hait, ce qui va permettre d'accroître la distance entre les deux. Nous remarquons ainsi que le début de la vie pulsionnelle est marqué par de petites violences acceptables à l'égard de l'objet. Toutefois le nourrisson comprend très tôt qu'il ne peut pas "trop dévorer sa mère", car il risque de la perdre et il apprend le plus souvent à maîtriser ce caractère destructeur de sa pulsion orale. La première interaction pulsionnelle engendre déjà un ratage de la satisfaction pleine des pulsions à l'égard de l'objet.

Dans la deuxième phase de l'organisation prégénitale, la phase sadique-anale, l'activité s'inscrit à travers la pulsion d'emprise. Plus précisément, le contenu intestinal traité comme une partie du corps propre représente "le premier cadeau" (Freud, 1905, p. 112) qui peut être retenu ou libéré. L'enfant se sert de cet autre objet - les excréments - dans la relation avec la personne qui prend soin de lui, en choisissant entre lui en donner ou pas. Le plaisir de faire ou de retenir l'objet est encore pour l'enfant synonyme du pouvoir d'une maîtrise sur l'objet. Comme l'observe Penot (2000), il s'agit d'un autre objet partiel détachable qui devra choir et qui sera représenté ultérieurement. De la même façon, on observe le caractère destructeur de la pulsion anale à l'égard de l'objet. Les excréments deviennent le moyen d'attaquer ou de contrôler la personne qui prend soin de l'enfant.

L'expérience spéculaire introduit deux autres objets dans la vie pulsionnelle: le regard et la voix. C'est à partir de cette identification avec l'image spéculaire extérieure, dans une relation duelle entre le moi et son double, que s'établit la première relation moi-objet. Cependant, l'image n'est pas suffisante pour cette 


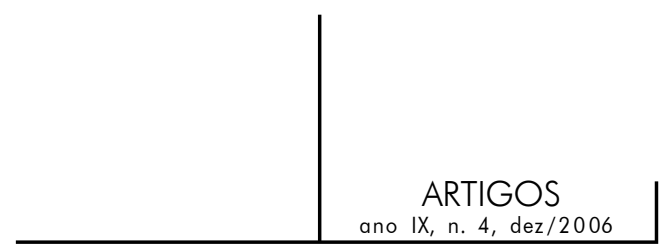

première identification. Il faut que deux autres objets soient présents pour accomplir l'identification à l'image. C'est la raison pour laquelle le regard et la voix de la mère, support du langage, jouent un rôle essentiel dans la relation imaginaire. La mère duplique le regard de l'enfant et sa voix lui donne le signe de reconnaissance de l'image. Dans ce jeu pulsionnel, le regard que la mère porte sur son enfant prouve aussi un affect sur ce qu'elle voit. En présence de la voix, la sonorité sur laquelle la mère émet les signes vient conférer ou communiquer le plaisir ou le déplaisir, donc la manifestation et l'intention de son propre désir.

Pour reprendre Freud (1905), la phase phallique est le temps où les aspirations sexuelles se dirigent vers une seule personne pour atteindre leurs buts. C'est devant l'absence du pénis que les enfants élaborent leurs théories dans cette phase. "Il y a bien un 'masculin' mais pas de féminin”, affirme Freud (1923, p. 116). Toutefois ce qui est présent à ce stade n'est pas la primauté de l’organe génital mais celle du phallus et de sa dimension imaginaire. En fait, le pénis se différencie des autres objets partiels dont il est l'héritier. Dans la croyance fantasmatique, autrement dit la menace de la castration, il est un objet qui peut venir à manquer mais qu'aucun objet réel ne peut représenter. Il devient pour l'enfant un objet privilégié du plaisir. La mère étant supposée avoir le manque du phallus, c'est cette place que le petit enfant revendique pour se faire aimer. Le père vient s'ajouter à cette dyade car il est celui qui peut donner le phallus à la mère. En fait, l'enfant doit se confronter à l'existence d'un père phallicisé imaginaire capable de satisfaire sa mère. La phase de l'organisation génitale infantile se met en place à travers la triangulation œdipienne avec comme point central le phallus. Pour l'enfant, le posséder est ce qui lui donne accès à la pleine jouissance. L'opération adolescente va être le temps de conclure que le phallus n'est pas un objet de possession du fait de sa place mythique.

Après la période de latence, qui permet d'édifier la barrière contre l'inceste, l'avènement pubertaire est le moment où le sujet va effectuer pleinement un choix d'objet. Si le complexe d'Edipe est ravivé à l'adolescence, cette période évoque également l'irruption pulsionnelle. Face au corps ayant subi des transformations, l'inceste devient possible et le mouvement pulsionnel réveillé conduit l'adolescent à la recherche d'autres objets hors de la famille. L'adolescent est donc obligé de déplacer l'investissement infantile qu'il a fait sur ses parents, premiers partenaires de l'interaction pulsionnelle.

L'adolescence se caractérise par la réactivation des pulsions partielles où l'individu doit surtout apprendre à limiter la force du mouvement de la pulsion destructrice. Autrement dit, il faut qu'il n'aille pas jusqu'au bout dans la réalisation de ses pulsions partielles, ce qui implique la reconnaissance de l'existence de l'autre, de l'objet comme autonome. Cette tâche qui a commencé à l'enfance doit s'accomplir à l'adolescence. Alors que les moyens de limitation et de maîtrise des 


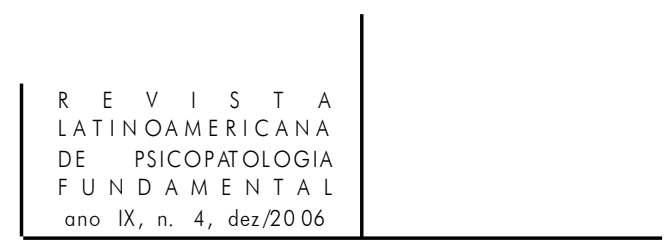

pulsions partielles sont testés progressivement au cours de l'enfance, l'adolescence est la mise à l'épreuve de la capacité de les sublimer, de les inhiber par un frein symbolique quelconque.

Cependant, limiter la réalisation des pulsions n'est pas une tâche si simple à l'adolescence. Pour preuve les changements du corps à cette période ; euxmêmes non maîtrisables, ils ne sont pas passibles d'être refusés par l'adolescent. Ce moment de l'irruption de l'espoir imaginaire du "tout sera possible quand je serai grand” est bouleversé par le réel pubertaire et entraîne aussi la désillusion : “j’ai grandi, je suis encore trop limité(e)”. Aucun objet de la réalité ne peut garantir la jouissance, l'illusion de la complétude du sujet.

Plus précisément, l'adolescent doit renoncer à la possession des objets de la pulsion de la prime enfance et à la jouissance de ces objets. Cette désillusion et les tentatives de déni sont au cœur de certaines pathologies à l'adolescence tel que la délinquance. De plus, l'objet réel visé par la pulsion, c'est-à-dire l'objet de la demande - le sein, la recherche d'un regard ou la quête d'un objet de la demande infantile - devra avoir laissé la place à une représentation, pendant le temps de la satisfaction, d'une marque inscrite comme signifiant de désir. L'énergie pulsionnelle sur la scène adolescente doit renvoyer non pas à un objet mais au désir lui-même, et le sujet devient désirant d'un désir et non d'un objet. L'adolescent va s'interroger sur sa place dans le registre du désir, dont la source émettrice a été le regard et la voix de l'Autre. En fait, l'adolescence va mettre à l'épreuve ce que l'Autre lui a communiqué sur l'intention de son propre désir.

\section{Les objets de la pulsion chez l'adolescent délinquant}

En fait, l'adolescent va rééditer les clichés aperçus et déjà fixés pendant son enfance. En ce qui concerne les objets de la pulsion, le type et l'intensité des liens de l'enfant avec ses objets déterminent l'orientation de ces liens pour la vie ultérieure. Partant de là, la cause première de la délinquance ou, pour reprendre Aichhorn (1951), l'état carentiel, doit être recherchée dans la petite enfance, où se sont établis "les premiers liens d'objets libidinaux déviant de la norme” (p. 110). Cette expression acceptée lors des premières phases du développement doit être dépassée à l'adolescence.

Ainsi, l'enfant qui grandit doit abandonner les objets libidinaux à l'intérieur de la famille. Lorsqu'il n'y parvient pas et qu'une fixation se met en place, la délinquance est souvent choisie comme issue. De manière plus précise, nous remarquons que des fixations trop intenses à certains membres de la famille, les premiers partenaires de l'interaction pulsionnelle, peuvent conduire à cette 


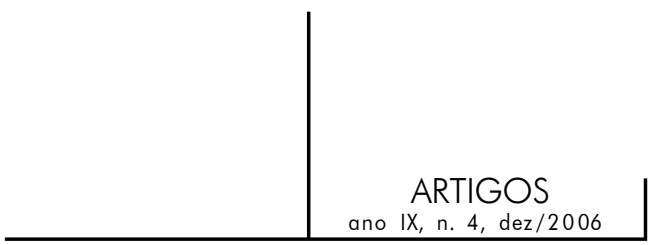

pathologie adolescente. Il semble que des fixations aux premiers objets de la pulsion rende la tâche de la puberté considérablement difficile. Certes, il n’a pas encore échoué, mais il cherche à accomplir cette tâche par une voie détournée.

La délinquance juvénile nous amène à concevoir que les forces en jeu sont celles des pulsions partielles. Ces pulsions qui devaient être imbriquées sous le primat du génital demeurent inchangées, restant sous l’égide de la phase prégénitale. L’événement de l'adolescence réactualise les pulsions dont le processus de sublimation est resté en suspens. L'adolescent est appelé à rechercher d’autres objets hors de la famille, avec pour objectif d’en prendre possession. La difficulté à réprimer des motions pulsionnelles et à les dévier de leurs buts primitifs est très souvent présente chez le jeune délinquant. Sur ce point, une ressemblance entre lui et l'enfant s'observe dans la mesure où, comme l'enfant, il poursuit des satisfactions pulsionnelles instantanées en ne parvenant pas à en changer le but originaire ni à abandonner un plaisir incertain pour l’obtenir plus tard.

Nous observons chez ces adolescents qu'une partie du Moi est dirigée par un principe de plaisir prédominant, dans le registre de la toute-puissance, et qu'une autre partie porte une maturité qui peut correspondre à la puberté. D’après Aichhorn (1951), tout jeune déviant présente ce clivage du Moi, c’est-à-dire ce “défaut d'évolution homogène du Moi” (p. 176). Nous pouvons penser que chez l'adolescent délinquant certaines phases de son développement affectif n'ont pas été dépassées correctement, ou que certaines fonctions psychiques opèrent à un stade évolutif inférieur.

L’appareil psychique est contraint de s’adapter aux conditions réelles qui apportent des difficultés s’opposant à la satisfaction des motions pulsionnelles, l'impossibilité de les satisfaire. Le déplaisir qui apparaît à chaque satisfaction d'un plaisir prohibé par la réalité non seulement occasionne le déplacement de gains de plaisir et le renoncement de ces gains mais en plus il suscite la répression des motions pulsionnelles. Ce remaniement se constituant au terme d'une évolution introduit la notion de l’objet visé par la pulsion comme autonome.

Ainsi, plus le principe de réalité se développe et s’intensifie, moins le Moi est livré sans défense à ses motions pulsionnelles. Il est manifeste que le principe de réalité se produit à partir des frustrations réelles qui suscitent une restriction pulsionnelle chez l'enfant tout en lui permettant de s'éloigner de l'objet. Cette tâche n'est pas si aisée, dans la mesure où il ne suffit pas de tenir compte des obstacles provenant du monde extérieur, il faut les éprouver et les retenir en tant que tels. Si la genèse du principe de réalité nécessite une certaine mesure de restriction des satisfactions pulsionnelles directes imposée par une frustration extérieure, l'excès ou le déficit de cette frustration extérieure empêchera la genèse de la restriction pulsionnelle nécessaire. Il ne fait aucun doute que cette fonction incombe en premier lieu à la mère. 


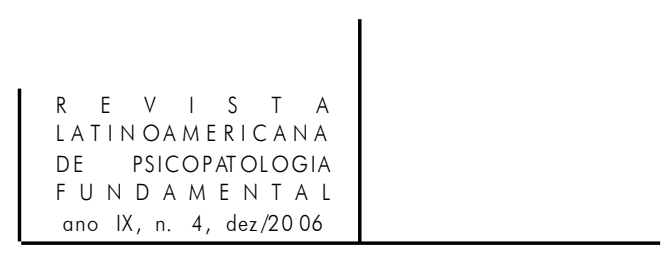

Pourtant, nous rencontrons souvent auprès des jeunes délinquants une mère qui les prend comme objets. Or, si le rôle des adultes pendant l'enfance est de poser l'interdit de prendre possession des objets qui appartiennent à l'autre, cet interdit ne peut s'établir quand la mère elle-même prend possession de l'enfant comme un objet partiel lui appartenant. Il n'est alors pas surprenant de voir comment ces adolescents prennent possession des objets. Dans l'histoire des jeunes délinquants, nous remarquons fréquemment une telle faiblesse de la mère lors du développement infantile, où elle ne réussissait pas à imposer les frustrations menant aux restrictions pulsionnelles nécessaires. Cette faiblesse se retrouve dans l'expérience spéculaire, et le regard et la voix de la mère vont être questionnés au moment de l'adolescence.

\section{Marcel: un Prométhée en possession du feu}

Marcel est âgé de quinze ans quand sa mère vient s'adresser au service de la clinique. De petite taille, mince et portant des vêtements de jeune fille, elle a l'air d'une adolescente lorsqu'elle se présente à la consultation. Marcel est le seul fils du couple, lequel a divorcé quand l'enfant était bébé. Avec un copain, il a mis le feu dans la salle de classe du lycée. La direction de l'établissement a appelé sa mère et lui a demandé de chercher un autre lycée pour son fils. Après discussions, le lycée accepte finalement de le laisser terminer son année scolaire, à condition qu'il engage une cure. Cet état de fait posait un premier problème puisqu'il ne s'agissait pas d'une demande mais d'une condition imposée par l'école.

La mère raconte l'incident en disant que son fils a été influencé par l'autre garçon et que la direction du lycée "avait quand même exagéré". Après la naissance de son fils elle a souffert d'une dépression, "ce qui l'a fait sentir une culpabilité de ne pas s'être occupée de son bébé comme il le fallait”. Concernant le père, elle nous apprend qu'ils ont divorcé quand Marcel avait neuf mois parce qu'il était alcoolique. Elle a fait en sorte que Marcel n'ait pas de contact avec son père, car selon elle "il lui fait du mal et il est nul". Il s'est remarié et ne boit plus depuis des années, pourtant c'est le parrain de Marcel, le mari de sa sœur, qui "a pris la place du père”. C'est toujours cet oncle maternel qu'elle inscrit comme père de Marcel sur la fiche d'inscription scolaire, et ce depuis son enfance. Les contacts de Marcel avec son père n’ont jamais été fréquents. La mère affirme qu'elle a évité les contacts pour le bien de son fils. Elle nous parle aussi de sa difficulté à poser des limites depuis que Marcel est adolescent. Elle s'enorgueillit d'une relation très proche avec son fils et raconte très fièrement "qu'il y a des gens qui pensent que nous sommes frère et sœur". 


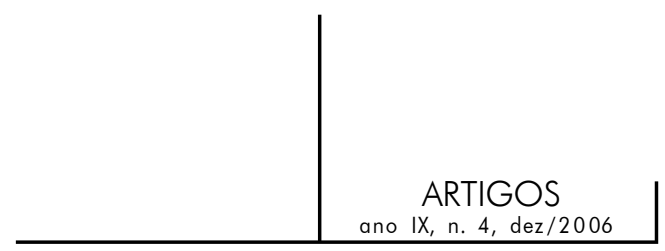

Marcel est un garçon d'un caractère docile et il semble très soumis. Au premier contact, il dit qu'il ne sait pas ce qu'il a fait et il ne sait même pas pourquoi il l'a fait. Il n'a pas pensé aux conséquences et ne voulait pas poser de problèmes à sa mère: “- Je ne voulais pas la faire souffrir, mais ça y est. Je n'ai pas pensé à ça avant”. Il ne sait pas comment raconter l'incident. C'est comme un moment d'inexistence, d'ailleurs couramment observable chez les adolescents délinquants au moment de l'agir. Le discours de Marcel illustre bien cette question: "- On a eu l'idée de mettre le feu dans la salle et c'est tout. Quand je me suis rendu compte, c'était fait. Tout de suite, j'ai pensé: Qu'est-ce que ma mère va dire ?" Marcel pleure en disant: “- Je ne sais pas pourquoi j'ai fait ça. Maintenant je dois quitter l'école de mon enfance..." Nous lui demandons si quelque chose s'est passé auparavant, mais il y répond par la négative.

Nous finissons par apprendre que peu de temps avant l'incident il avait appris que son parrain divorçait. La veille de l'incendie était le jour de la semaine où il avait l'habitude de jouer au tennis avec son parrain. Mais celui-ci n'est pas venu le chercher et sa mère lui avait laissé entendre que peut-être ils ne joueraient plus ensemble. "- Mais ça n'a aucune importance", souligne-t-il.

Il est manifeste que ce garçon a perdu l'espoir d'un tiers séparateur, le parrain inscrit à la place du Nom-du-Père, le seul autorisé par la mère. D'autre part, en incendiant l'école de son enfance Marcel se fait brûler dans la scène réelle, en dehors de son corps. Il fait disparaître une partie de lui-même, détruit une partie de son enfance - l'école, représentante de son enfance et des premiers objets dont il n'arrive pas à se séparer - pour provoquer un manque. L'éloignement du parrain vient déclencher l'agir car l'exclusion de cette personne, qui occupait d'une certaine façon la place de tiers entre lui et sa mère, le renvoie à nouveau à l'infantile, à la liaison à la Mère archaïque.

Quelques jours plus tard, c'est son père qui demande à être reçu. Il est très inquiet et se plaint de ne pas avoir su plus tôt ce qui s'était passé au lycée. Il se plaint aussi d'être éloigné de son fils. Juste après le divorce, il a insisté pour maintenir le contact mais Marcel était trop petit et sa mère ne voulait pas le laisser sortir. Depuis quelque temps ils se revoient, mais pas fréquemment. Il évoque son passé alcoolique et la cure de désintoxication qu'il a suivie. Depuis quelques années il ne boit plus mais il est "devenu un peu exigeant".

Marcel reprend des contacts un peu plus réguliers avec son père. Il quittera le lycée à la fin de l'année et s'inscrira dans un autre, avec cette fois le nom du père sur la fiche d'inscription. Trois mois après la rentrée de la nouvelle année scolaire, Marcel met à nouveau le feu au lycée, dans la cour. Il est clair qu'on ne peut attendre de l'adolescent qu'il arrête aussitôt de privilégier des conduites agies. Au contraire, il se peut qu'il agisse ainsi jusqu'à ce qu'il puisse accéder à la parole à la place de l'agir. 


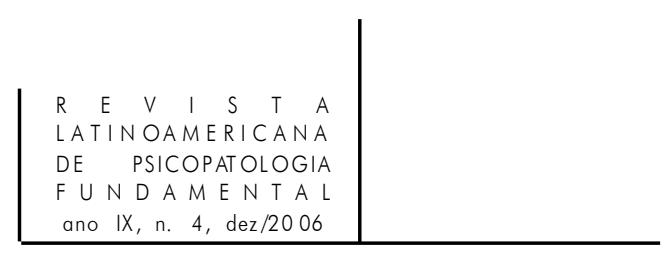

En fait, Marcel n'arrive pas à maîtriser autrement les pulsions libidinales et destructrices. Il ne fait que matérialiser ses fantasmes dans la réalité extérieure parce qu'il ne trouve pas de supports internes pour le faire d'une autre manière. La haine en tant que moyen de préservation du lien érotique inconscient ne peut se montrer qu'à travers cette conduite car, comme il nous le dit, "il ne voulait pas faire souffrir sa mère".

Apparaît aussi la question de l'Autre maternel. Marcel se demande d'emblée ce que sa mère lui dirait. Cependant, il se place dans une position dépressive après ce second incident. Il commence à se poser des questions sur la relation avec sa mère et nous pouvons y travailler ensemble. Il se demande pourquoi il doit s'assujettir au désir de sa mère, et donne l'exemple d'un cours d'informatique qu'elle lui a fait commencer. Il a toujours accepté ce que sa mère lui proposait.

La fragilité des parents est bien réelle, aussi bien de la mère qui ne peut pas supporter la séparation que du père qui se protège de l'alcoolisme derrière des exigences. En outre, la haine n'est pas consciente pour l'éloigner de sa mère. Il faut donc la faire apparaître dans des objets de la réalité et dans la réalité extérieure en faisant disparaître le sujet de l'acte.

Par rapport à la prise de possession du feu, Freud (1932) établit son hypothèse en parlant des hommes des origines et la confirme en suivant l'interprétation de la légende grecque de Prométhée. Freud prend les traits qui autorisent une interprétation analytique tout en prenant en compte les déformations prévisibles: la façon dont Prométhée transporte le feu, le caractère de l'acte et le sens de son châtiment. Prométhée, héros culturel, apporte aux hommes le feu qu'il a dérobé aux dieux, caché dans un bâton creux, une tige de fenouil.

La question posée par Freud est très pertinente: "pourquoi la prise de possession du feu est-elle inséparablement liée à la représentation d'un délit?" (p. 193) Dans le partage des offrandes, Prométhée désavantage Zeus en faveur des hommes et donc ce sont les dieux qui sont trompés. Le mythe qui attribue aux dieux la satisfaction de toutes les convoitises auxquelles le petit d'homme doit renoncer - comme l'inceste par exemple, observe Freud (1932) - "est suffisamment connu. En termes analytiques, la vie pulsionnelle, le Ça, est le dieu trompé par le renoncement à étendre le feu” (p. 194).

Un autre trait de la légende et le renversement en son contraire est le plus important: le châtiment du Porteur du feu. Prométhée reste enchainé à un rocher et un vautour vient chaque jour lui dévorer le foie. Rappelons que cet organe était considéré par les Anciens comme le siège de toutes les passions et de tous les désirs. C’est pourquoi le châtiment de Prométhée serait le plus approprié pour un criminel poussé par ses pulsions et qui a commis un acte sur impulsion de ses convoitises. De plus, dans la légende de Prométhée ainsi que dans d'autres mythes sur le feu, ce dernier apparaît nécessairement comme analogue à la passion 


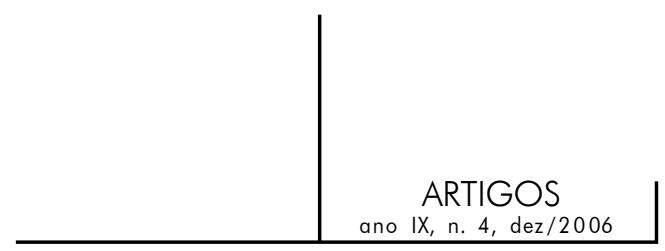

amoureuse, c'est-à-dire comme symbole de la libido. Nous pouvons entendre cela comme la chaleur qui irradie du feu et qui provoque la même sensation que celle de l'état d'excitation sexuelle. De même, la flamme évoque de par sa forme et ses mouvements le phallus en activité.

Le cas de Marcel montre distinctement que la relation à la mère se constitue de dérapages incestueux desquels la haine n’arrive pas à le protéger. Au contraire, à chaque fois qu'il entre en conflit avec sa mère, elle se montre extrêmement fragilisée. Le but de la relation aux premiers objets de la pulsion demeure inchangé et il n’arrive pas à la maîtriser. Lorsqu'il ne trouve pas de supports internes suffisants pour se donner des représentations, il matérialise le fantasme ; autrement dit, il prend possession du feu, le représentant de sa libido en dehors de son corps.

\section{Conclusion}

L’adolescent délinquant est régi par un principe de plaisir non dépassé et dans lequel les relations aux premiers objets de la pulsion spécifique de l'enfance, eux aussi non liquidés normalement, jouent un rôle. De fait, ce jeune cherche impulsivement la satisfaction de ses pulsions les plus primitives, d'une façon purement automatique. De même, la caractéristique de l’objet de la délinquance juvénile est d'être un objet de la réalité. C’est donc à travers les objets de réalité que ces adolescents révèlent leur rapport intime à l’objet psychique. Comme ce jeune ne trouve pas de limites solides entre dedans et dehors, entre sujet et objet, la réalité externe lui sert à établir un système de communication dont il attend un message qui puisse ordonner ses désirs.

\section{Références}

AichHorn, A. (1951). Jeunes en souffrance: psychanalyse et éducation spécialisée. Lecques: Les éditions du Champ Social, 2000.

Freud, S. (1905). Trois essais sur la théorie sexuelle. Trad. fr. P. Koeppel. Paris: Gallimard, 1978.

(1915). Pulsions et destins des pulsions. In: Métapsychologie (1946). Trad. fr. J. Laplanche, J.-B. Pontalis. Paris: Gallimard, 1968. p. 11-43.

(1923). L’organisation génitale infantile. In: La vie sexuelle. Trad. fr. D. Berger, J. Laplanche. Paris: PUF, 1977. p. 113-6. 


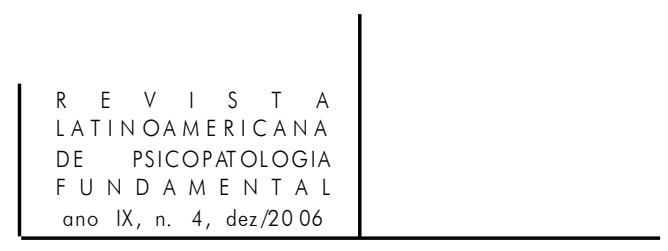

(1932). Sur la prise de possession du feu. In: Résultats, idées, problèmes II (1921-1938). Trad. fr. J. Laplanche, J. Sédat. Paris: PUF, 1995. p. 191-6. 1949.

(1938). Abrégé de psychanalyse. Trad. fr. A. Berman, J. Laplanche. Paris: PUF,

LACAN, J. (1959-1960). Le séminaire. Livre VII. L'éthique de la psychanalyse. Paris: Seuil, 1986.

Penot, B. La passion du sujet freudien: entre pulsionnalité et signifiance. Ramonville Saint-Agne: Érès, 2000.

\section{Resumés}

Ao longo do desenvolvimento infantil, o ser humano deve se formar representações dos objetos da pulsão no interior da psique. A adolescência não faz senão mostrar os efeitos das primeiras experiências vividas, em particular as possibilidades de se construir um objeto psíquico. A dialética entre o dentro e o fora, entre sujeito e objeto inscrita com os primeiros objetos da pulsão está no centro da patologia da delinquência juvenil.

Palavras-chave: Objetos da pulsão, objeto psíquico, adolescência, delinqüência

A lo largo del desenvolvimiento infantil, el ser humano debe se formar representaciones de los objetos de la pulsión en el interior de la psique. La adolescencia no hace más que mostrar los efectos de las primeras experiencias vividas, en particular las posibilidades de construcción de un objeto psíquico. La dialéctica entre el dentro y el fuera, entre sujeto y objeto inscrita con los primeros objetos de la pulsión, está en el centro de la patología de la delincuencia juvenil.

Palabras claves: Objetos de la pulsión, objeto psíquico, adolescencia, delincuencia

Throughout his or her childhood development a human being must formulate representations of drive objects inside the mental apparatus. Adolescence merely shows the effects of the earliest experiences, especially the possibility to construct oneself as a psychic object. The dialectic between the inside and the outside, between subject and object inscribed with the first objects of the drive is at the center of the pathology of juvenile delinquency.

Key words: Objects of the drives, psychic object, adolescence, delinquency

Versão inicial recebida em abril de 2006

Versão revisada recebida em julho de 2006 